\title{
Periodicity of the Positive Solutions of a Fuzzy Max-Difference Equation
}

\author{
Qiuli He, ${ }^{1,2}$ Chunyan Tao, ${ }^{3}$ Taixiang Sun, ${ }^{2,3}$ Xinhe Liu, ${ }^{3}$ and Dongwei Su ${ }^{1,2}$ \\ ${ }^{1}$ College of Electrical Engineering, Guangxi University, Nanning, Guangxi 530004, China \\ ${ }^{2}$ Guangxi Key Laboratory of Power System Optimization and Energy Technology, Nanning, Guangxi 530004, China \\ ${ }^{3}$ College of Mathematics and Information Science, Guangxi University, Nanning, Guangxi 530004, China
}

Correspondence should be addressed to Taixiang Sun; stx1963@163.com

Received 20 March 2014; Accepted 13 April 2014; Published 22 May 2014

Academic Editor: Tongxing Li

Copyright (c) 2014 Qiuli He et al. This is an open access article distributed under the Creative Commons Attribution License, which permits unrestricted use, distribution, and reproduction in any medium, provided the original work is properly cited.

We investigate the periodic nature of the positive solutions of the fuzzy max-difference equation $x_{n+1}=\max \left\{A_{n} / x_{n-m}, x_{n-k}\right\}, n=$ $0,1, \ldots$, where $k, m \in\{1,2, \ldots\}, A_{n}$ is a periodic sequence of fuzzy numbers, and $x_{-d}, x_{-d+1}, \ldots, x_{0}$ are positive fuzzy numbers with $d=\{m, k\}$. We show that every positive solution of this equation is eventually periodic with period $k+1$.

\section{Introduction}

The max operator arises naturally in certain models in automatic control theory (see $[1,2])$. In recent years, the discrete case involving difference equations with maximum has been receiving increasing attention (see [3-8]). Elsayed and Stević [9] considered the max-difference equation

$$
x_{n+1}=\max \left\{\frac{B}{x_{n}}, x_{n-2}\right\}, \quad n=0,1, \ldots,
$$

where $B \in \mathbf{R} \equiv(-\infty,+\infty)$ and the initial conditions $x_{-2}, x_{-1}, x_{0} \in \mathbf{R}$ and showed that every well-defined solution of this equation is eventually periodic with period 3 .

In [10], Iričanin and Elsayed investigated the maxdifference equation

$$
x_{n+1}=\max \left\{\frac{B}{x_{n}}, x_{n-3}\right\}, \quad n=0,1, \ldots,
$$

where $B \in \mathbf{R}$ and the initial conditions $x_{-3}, x_{-2}, x_{-1}, x_{0} \in \mathbf{R}$ and showed that every well-defined solution of this equation is eventually periodic with period 4 .

Recently Xiao and Shi [11] studied the max-difference equation

$$
x_{n+1}=\max \left\{\frac{B}{x_{n}}, x_{n-1}\right\}, \quad n=0,1, \ldots,
$$

where $B \in \mathbf{R}$ and the initial conditions $x_{-1}, x_{0} \in \mathbf{R}$ and showed that every well-defined solution of the above equation is eventually periodic with period 2 .

In [12], we dealt with the max-difference equation

$$
x_{n+1}=\max \left\{\frac{B}{x_{n}}, x_{n-k}\right\}, \quad n=0,1, \ldots,
$$

where $B \in \mathbf{R}, k \in\{1,2, \ldots\}$ and the initial conditions $x_{-k}, x_{-k+1}, \ldots, x_{0} \in \mathbf{R}$ and showed that every well-defined solution of the above equation is eventually periodic with period $k+1$, which extended the results of [9-11] to the general case.

Recently there has been an increase in interest in the study of fuzzy difference equations (see [13-15]). In [16], Stefanidou and Papaschinopoulos studied the periodicity of the positive solutions of the following fuzzy max-difference equation

$$
x_{n}=\max \left\{\frac{A}{x_{n-k}}, \frac{B}{x_{n-m}}\right\}, \quad n=0,1, \ldots
$$

where $A, B$, and the initial conditions $x_{-d}, x_{-d+1}, \ldots, x_{0}$ with $d=\max \{k, m\}$ are positive fuzzy numbers. 
In [17], Zhang et al. dealt with the existence, the boundedness, and the asymptotic behavior of the positive solutions to a first order fuzzy Ricatti difference equation

$$
x_{n+1}=\frac{A+x_{n}}{B+x_{n}}, \quad n=0,1, \ldots,
$$

where $A, B$, and the initial condition $x_{0}$ are positive fuzzy numbers.

In this note, our goal is to investigate the periodicity of the positive solutions of the fuzzy max-difference equation

$$
x_{n+1}=\max \left\{\frac{A_{n}}{x_{n-m}}, x_{n-k}\right\}, \quad n=0,1, \ldots,
$$

where $k, m \in\{1,2, \ldots\}, A_{n}$ is a periodic sequence of fuzzy numbers, and $x_{-d}, x_{-d+1}, \ldots, x_{0}$ are positive fuzzy numbers with $d=\{m, k\}$. Our main result is the following theorem.

Theorem 1. Let $k, m \in\{1,2, \ldots\}$ and $A_{n}$ be a periodic sequence of fuzzy numbers. Then every positive solution of (7) is eventually periodic with period $k+1$.

\section{Preliminaries}

We need the following definitions. A function $U$ from $\mathbf{R}^{+}=$ $(0,+\infty)$ into the interval $[0,1]$ is called a fuzzy number if the following statements hold (see [18]).

(1) $U$ is normal (i.e., $U(x)=1$ for some $x \in \mathbf{R}^{+}$).

(2) $U$ is a convex fuzzy set (i.e., $U(\lambda x+(1-\lambda) y) \geq$ $\min \{U(x), U(y)\}$ for any $\lambda \in[0,1]$ and any $x, y \in$ $\mathbf{R}^{+}$).

(3) $U$ is upper semicontinuous.

(4) The support supp $U=\overline{\bigcup_{a \in(0,1]}[U]_{a}}=\overline{\{x: U(x)>0\}}$ is compact,

where $[U]_{a}=\left\{x \in \mathbf{R}^{+}: U(x) \geq a\right\}$ (for any $a \in(0,1]$ ) (which are said to be the $a$-cuts of the fuzzy number $U$ ) and $\bar{M}$ is the closure of set $M$. We see from [19, Theorem 3.1.5 and Theorem 3.1.8] the $a$-cuts of the fuzzy number $U$ are closed intervals.

A fuzzy number $U$ is said to be positive if $\min (\operatorname{supp} U)>$ 0 . If $U \in \mathbf{R}^{+}$, then $U$ is a positive fuzzy number (it is called a trivial fuzzy number also) with $[U]_{a}=[U, U]$ for any $a \in$ $(0,1]$.

For some positive integer $k$, let $U_{1}, U_{2}, \ldots, U_{k}$ be fuzzy numbers and $a \in(0,1]$ with

$$
\left[U_{i}\right]_{a}=\left[U_{i, l, a}, U_{i, r, a}\right] \text { for } 0 \leq i \leq k .
$$

Write

$$
\begin{aligned}
& V_{l, a}=\max \left\{U_{i, l, a}: 0 \leq i \leq k\right\}, \\
& V_{r, a}=\max \left\{U_{i, r, a}: 0 \leq i \leq k\right\} .
\end{aligned}
$$

Then we know from [20, Theorem 2.1] that there exists a fuzzy number $V$ such that

$$
[V]_{a}=\left[V_{l, a}, V_{r, a}\right] \quad \text { for any } a \in(0,1] .
$$

By [21] and [22, Lemma 2.3] one can define

$$
V=\max \left\{U_{i}: 0 \leq i \leq k\right\}
$$

A sequence of positive fuzzy numbers $\left\{x_{n}\right\}_{n=-d}^{\infty}$ is said to be a solution of (7) if it satisfies (7). If there exists a positive integer $M$ and $p$ such that, for all $n \geq M$,

$$
x_{n+p}=x_{n}
$$

then $\left\{x_{n}\right\}_{n=-d}^{\infty}$ is said to be eventually periodic with period $p$.

Proposition 2. Let $x_{-d}, x_{-d+1}, \ldots, x_{0}$ be a sequence of positive fuzzy numbers. Then there exists a unique positive solution $\left\{x_{n}\right\}_{n=-d}^{\infty}$ of (7) with initial values $x_{-d}, x_{-d+1}, \ldots, x_{0}$.

Proof. Assume that $\left[A_{n}\right]_{a}=\left[A_{n, l, a}, A_{n, r, a}\right]$ (for any $a \in(0,1]$ ) and $n \geq 0$. Let $x_{-d}, x_{-d+1}, \ldots, x_{0}$ be positive fuzzy numbers such that

$$
\left[x_{i}\right]_{a}=\left[P_{i, a}, Q_{i, a}\right] \quad \text { for }-d \leq i \leq 0, a \in(0,1]
$$

and let $\left\{\left(P_{n, a}, Q_{n, a}\right)\right\}_{n=-d}^{\infty}(a \in(0,1])$ be the unique positive solution of the following system of difference equations:

$$
\begin{gathered}
P_{n+1, a}=\max \left\{\frac{A_{n, l, a}}{Q_{n-m, a}}, P_{n-k, a}\right\}, \\
Q_{n+1, a}=\max \left\{\frac{A_{n, r, a}}{P_{n-m, a}}, Q_{n-k, a}\right\},
\end{gathered}
$$

with initial values $\left(P_{i, a}, Q_{i, a}\right)(-d \leq i \leq 0)$. Arguing as in Proposition 3.1 of [23] we may show that $\left\{\left(P_{n, a}, Q_{n, a}\right)\right\}_{n=-d}^{\infty}(a \in$ $(0,1])$ determines a sequence of positive fuzzy numbers $\left\{x_{n}\right\}_{n=-d}^{\infty}$ with

$$
\left[x_{n}\right]_{a}=\left[P_{n, a}, Q_{n, a}\right], \quad n \geq-d, a \in(0,1],
$$

and that $\left\{x_{n}\right\}_{n=-d}^{\infty}$ is the unique positive solution of (7) with initial values $x_{-d}, x_{-d+1}, \ldots, x_{0}$. This completes the proof of the proposition.

\section{Proof of Theorem 1}

Lemma 3. Consider the system of difference equations

$$
\begin{aligned}
& y_{n+1}=\max \left\{\frac{C_{n}}{z_{n-m}}, y_{n-k}\right\}, \\
& z_{n+1}=\max \left\{\frac{B_{n}}{y_{n-m}}, z_{n-k}\right\}, \quad n=0,1, \ldots,
\end{aligned}
$$

where $B_{n}, C_{n}$ are two periodic sequences of positive real numbers and the initial values $y_{-d}, z_{-d}, \ldots, y_{0}, z_{0}$ are positive real numbers. Then every positive solution of (16) is eventually periodic of period $k+1$. 
Proof. Let $\left\{\left(y_{n}, z_{n}\right)\right\}_{n=-d}^{\infty}$ be a positive solution of (16). We have from (16) that, for any $n \geq 0$ and any $i \geq 0$,

$$
\begin{aligned}
& y_{(n+1)(k+1)+i} \\
& \quad=\max \left\{\frac{C_{(n+1)(k+1)+i-1}}{z_{(n+1)(k+1)+i-m-1}}, y_{n(k+1)+i}\right\} \geq y_{n(k+1)+i}, \\
& z_{(n+1)(k+1)+i} \\
& \quad=\max \left\{\frac{B_{(n+1)(k+1)+i-1}}{y_{(n+1)(k+1)+i-m-1}}, z_{n(k+1)+i}\right\} \geq z_{n(k+1)+i} .
\end{aligned}
$$

Then $\left\{y_{n(k+1)+i}\right\}_{n=0}^{\infty}$ and $\left\{z_{n(k+1)+i}\right\}_{n=0}^{\infty}$ are increasing for every $0 \leq i \leq k$.

Now we show that $\left\{y_{n(k+1)+i}\right\}_{n=0}^{+\infty}$ is a constant sequence eventually for every $0 \leq i \leq k$. Indeed, if $\left\{y_{n(k+1)+r}\right\}_{n=0}^{+\infty}$ is not constant sequence eventually for some $0 \leq r \leq k$, then there exist $k m<n_{1}<n_{2}<\cdots$ such that $y_{n_{i}(k+1)+r}>y_{\left(n_{i}-1\right)(k+1)+r}$ and $C_{n_{i}(k+1)+r-1}$ is a constant sequence for all $i \geq 1$ since $C_{n}$ is a periodic sequence. Thus we have

$$
\begin{aligned}
& y n_{i+1}(k+1)+r \\
& =\max \left\{\frac{C_{n_{i+1}(k+1)+r-1}}{z_{n_{i+1}(k+1)+r-m-1}}, y_{\left(n_{i+1}-1\right)(k+1)+r}\right\} \\
& =\frac{C_{n_{i+1}(k+1)+r-1}}{z_{n_{i+1}(k+1)+r-m-1}}>y_{\left(n_{i+1}-1\right)(k+1)+r} \\
& \geq y_{n_{i}(k+1)+r}=\max \left\{\frac{C_{n_{i}(k+1)+r-1}}{z_{n_{i}(k+1)+r-m-1}}, y_{\left(n_{i}-1\right)(k+1)+r}\right\} \\
& =\frac{C_{n_{i}(k+1)+r-1}}{z_{n_{i}(k+1)+r-m-1}} .
\end{aligned}
$$

From this we obtain that, for all $i \geq 1$,

$$
z_{n_{i}(k+1)+r-m-1}>z_{n_{i+1}(k+1)+r-m-1} .
$$

This is a contradiction.

In a similar fashion, we can show that $\left\{z_{n(k+1)+i}\right\}_{n=0}^{+\infty}$ is also a constant sequence eventually for every $0 \leq i \leq k$.

From the above we see that $\left\{\left(y_{n}, z_{n}\right)\right\}_{n=-d}^{\infty}$ is eventually periodic with period $k+1$. This completes the proof of Lemma 3.

Proof of Theorem 1. Let $\left\{x_{n}\right\}_{n=-d}^{\infty}$ be a positive solution of (7) with initial values $x_{-d}, x_{-d+1}, \ldots, x_{0}$ satisfying (13) and let (15) hold. We see from Proposition 2 that $\left\{\left(P_{n, a}, Q_{n, a}\right)\right\}_{n=-d}^{\infty}(a \in$ $(0,1])$ satisfies system (14). Using Lemma 3 we know that $\left\{\left(P_{n, a}, Q_{n, a}\right)\right\}_{n=-d}^{\infty}$ is eventually periodic with period $k+1$. Therefore, it follows from (14) and Lemma 3 that $\left\{x_{n}\right\}_{n=-d}^{\infty}$ is eventually periodic of period $k+1$. This completes the proof of Theorem 1.

\section{Conflict of Interests}

The authors declare that there is no conflict of interests regarding the publication of this paper.

\section{Acknowledgments}

This project is supported by NNSF of China (11261005) and NSF of Guangxi (2012GXNSFDA276040).

\section{References}

[1] A. D. Mishkis, "On some problems of the theory of differential equations with deviating argument," Uspekhi Matematicheskikh Nauk, vol. 32, no. 2, pp. 173-202, 1977.

[2] E. P. Popov, Automatic Regulation and Control, Nauka, Moscow, Russia, 1966 (Russian).

[3] S. Stević, "On a symmetric system of max-type difference equations," Applied Mathematics and Computation, vol. 219, no. 15, pp. 8407-8412, 2013.

[4] S. Stević, "On positive solutions of some classes of max-type systems of difference equations," Applied Mathematics and Computation, vol. 232, pp. 445-452, 2014.

[5] S. Stević, M. A. Alghamdi, A. Alotaibi, and N. Shahzad, "Eventual periodicity of some systems of max-type difference equations," Applied Mathematics and Computation, vol. 236, pp. 635-641, 2014.

[6] T. Sun, B. Qin, H. Xi, and C. Han, "Global behavior of the maxtype difference equation $x_{n+1}=\max \left\{1 / x_{n}, A_{n} / x_{n-1}\right\}$," Abstract and Applied Analysis, vol. 2009, Article ID 152964, 10 pages, 2009.

[7] T. Sun, H. Xi, C. Han, and B. Qin, "Dynamics of the max-type difference equation $x_{n}=\max \left\{1 / x_{n-m}, A_{n} / x_{n-r}\right\}$," Journal of Applied Mathematics and Computing, vol. 38, no. 1-2, pp. 173180, 2012.

[8] T. Sun, H. Xi, and B. Qin, "Global behavior of the max-type difference equation $x_{n+1}=\max \left\{A / x_{n-m}, 1 / x_{n-k}^{\alpha}\right\}$," Journal of Concrete and Applicable Mathematics, vol. 10, no. 1-2, pp. 32-39, 2012.

[9] E. M. Elsayed and S. Stević, "On the max-type equation $x_{n+1}=\max \left\{A / x_{n}, x_{n-2}\right\}$," Nonlinear Analysis: Theory, Methods \& Applications A: Theory and Methods, vol. 71, no. 3-4, pp. 910922, 2009.

[10] B. D. Iričanin and E. M. Elsayed, "On the max-type difference equation $x_{n+1}=\max \left\{A / x_{n}, x_{n-3}\right\}$," Discrete Dynamics in Nature and Society, vol. 2010, Article ID 675413, 13 pages, 2010.

[11] Q. Xiao and Q.-h. Shi, "Eventually periodic solutions of a maxtype equation," Mathematical and Computer Modelling, vol. 57, no. 3-4, pp. 992-996, 2013.

[12] B. Qin, T. Sun, and H. Xi, "Dynamics of the max-type difference equation $x_{n+1}=\max \left\{A / x_{n}, x_{n-k}\right\}$," Journal of Computational Analysis and Applications, vol. 14, no. 5, pp. 856-861, 2012.

[13] Q. H. Zhang and J. Z. Liu, “The first-order fuzzy difference equation $x_{n+1}=A x_{n}+B$," Fuzzy Systems and Mathematics, vol. 23, no. 4, pp. 74-79, 2009 (Chinese).

[14] Q. Zhang, L. Yang, and D. Liao, "On the fuzzy difference equation $x_{n+1}=A+\sum_{i=0}^{k} B / x_{n-i}$," World Academy of Science, Engineering and Technology, vol. 75, pp. 1032-1037, 2011.

[15] Q. H. Zhang, L. H. Yang, and D. X. Liao, "Behavior of solutions to a fuzzy nonlinear difference equation," Iranian Journal of Fuzzy Systems, vol. 9, no. 2, pp. 1-12, 2012.

[16] G. Stefanidou and G. Papaschinopoulos, "The periodic nature of the positive solutions of a nonlinear fuzzy max-difference equation," Information Sciences, vol. 176, no. 24, pp. 3694-3710, 2006. 
[17] Q. Zhang, L. Yang, and D. Liao, "On first order fuzzy Ricatti difference equation," Information Sciences, vol. 270, pp. 226236, 2014.

[18] G. Papaschinopoulos and B. K. Papadopoulos, "On the fuzzy difference equation $x_{n+1}=A+x_{n} / x_{n-m}$," Fuzzy Sets and Systems, vol. 129, no. 1, pp. 73-81, 2002.

[19] H. T. Nguyen and E. A. Walker, A First Course in Fuzzy Logic, CRC Press, Boca Raton, Fla, USA, 1997.

[20] C. Wu and B. Zhang, "Embedding problem of noncompact fuzzy number space $E^{-}(\mathrm{I})$," Fuzzy Sets and Systems, vol. 105, no. 1, pp. 165-169, 1999.

[21] G. J. Klir and B. Yuan, Fuzzy Sets and Fuzzy Logic, Prentice Hall PTR, Upper Saddle River, NJ, USA, 1995.

[22] G. Papaschinopoulos and B. K. Papadopoulos, "On the fuzzy difference equation $x_{n+1}=A+B / x_{n}$," Soft Computing, vol. 6, pp. 456-461, 2002.

[23] G. Stefanidou and G. Papaschinopoulos, "Behavior of the positive solutions of fuzzy max-difference equations," Advances in Difference Equations, no. 2, pp. 153-172, 2005. 


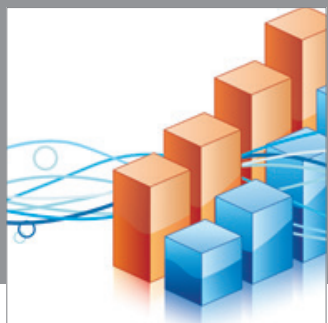

Advances in

Operations Research

mansans

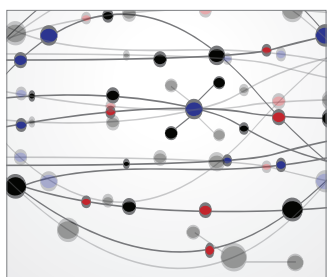

The Scientific World Journal
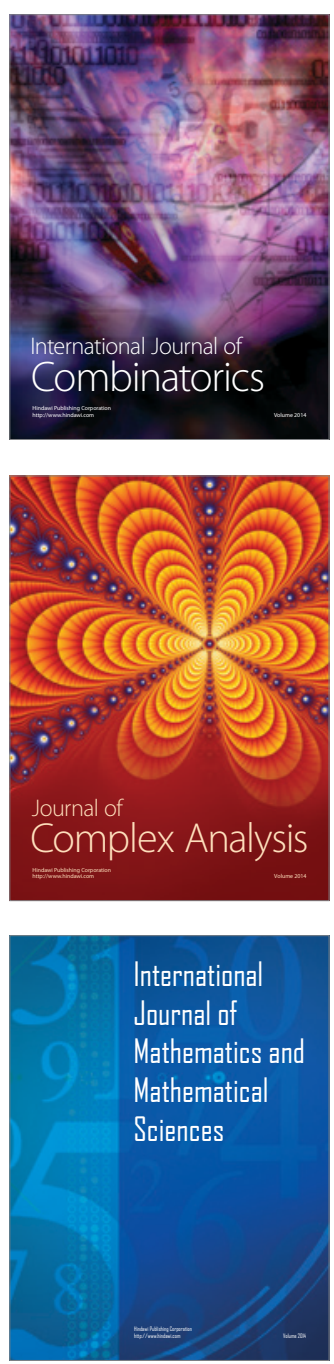
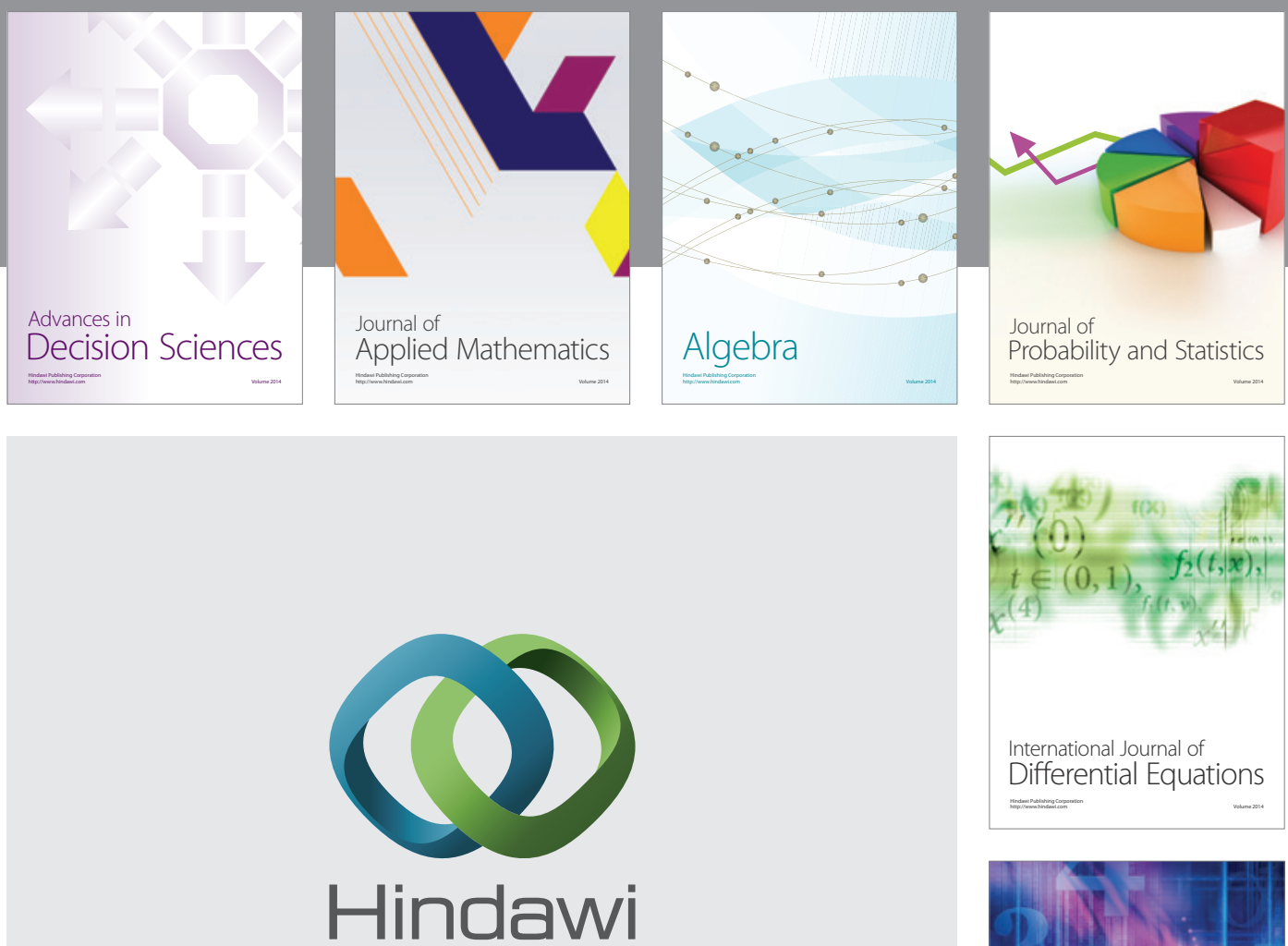

Submit your manuscripts at http://www.hindawi.com
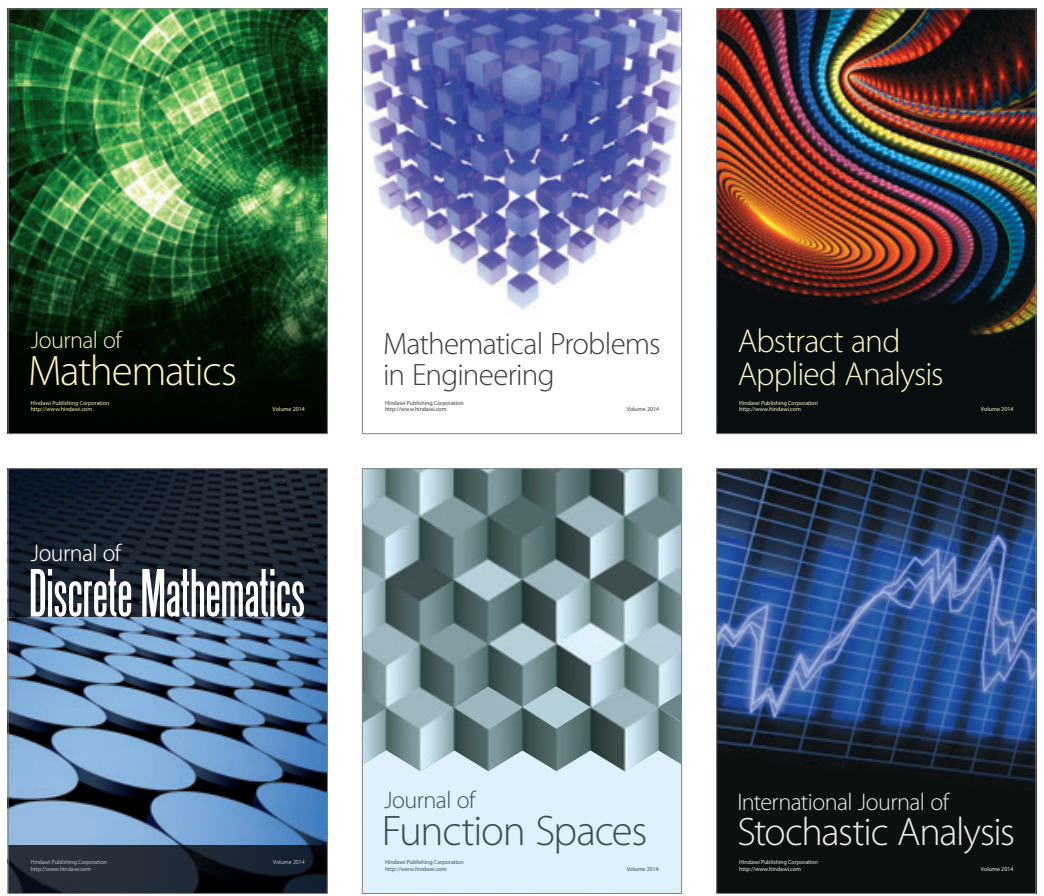

Journal of

Function Spaces

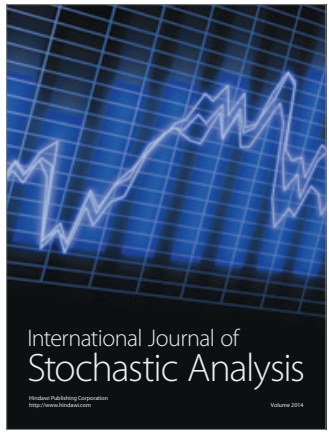

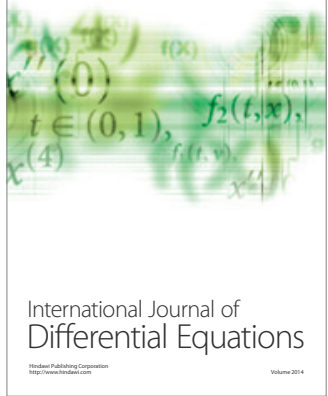
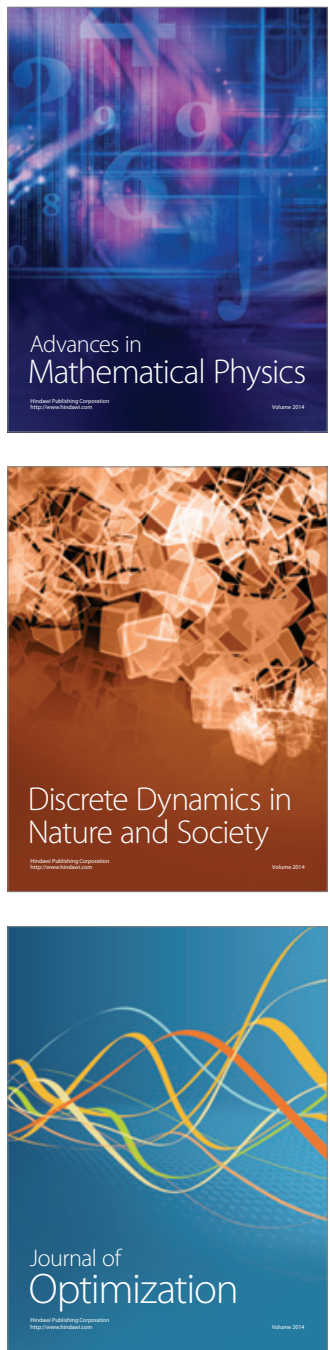